\title{
A Modified Node Selection Scheme for Cooperative Wireless Networks
}

\author{
S.Sadasivam \\ Associate professor \\ Sethu institute of technology \\ Pulloor, India
}

\author{
M.Sheik Dawood \\ Associate professor \\ Sethu institute of technology \\ Pulloor, India
}

\author{
G.Athisha \\ Professor \\ PSNA College of Engg. \&tech \\ Dindigul, India
}

\begin{abstract}
Cooperative relaying is an emerging technology that can compensate for the effects of multipath fading and shadowing by employing multiple relay nodes between sources and destinations. It has the potential to provide reliable transmission, high throughput, and broad coverage, thus drawing great attention in recent years. This paper proposes a node selection scheme in cooperative wireless networks. In a single-hop network with multiple relays, selecting a single node to aid in the transmission between a source and a destination outperforms both traditional orthogonal transmissions and distributed space-time codes. The usage of multiple hops will further improve the wireless communication. In wireless networks where transmitter transmits data over multiple hops and relays can communicate with each other, the relationship between cooperation and channel-adaptive routing is provided in this paper. The cooperation is only helpful if designed combine with a routing technique. However, the routing scheme used for combining with cooperation is very complex and involves large computation overhead. To overcome these problems, optimized link state routing is implemented in this paper. The simulation result shows that the proposed node selection scheme will results in better selection of nodes for cooperative wireless networks.
\end{abstract}

\section{Index Terms}

Cooperative networks, Cooperative routing.

\section{Keywords}

Node Selection Distributed Space-Time Coding, Optimized Link State Routing.

\section{INTRODUCTION}

With the advent of new technologies and the demand for flexibility and ease in working environment, the use of mobile wireless computing is growing fast. Besides their use, mobile wireless networks are assumed to grow in size too. They can function in independent groups, containing some tens of nodes up to several hundreds of nodes. As the network size increases, it becomes common for the nodes to bedispersed in a larger area than the radio range of individual nodes. Under such conditions, one has to employ routing techniques such that the out of range nodes may communicate with each other via intermediate nodes. The constructions of wireless networks face the challenge of increasing data rates to satisfy the needs of future generations of networks. The signal at the transmitter, however, suffers from channel fading caused by the super positioning of multiple rejected and refracted copies of a signal arriving from different directions. The destination therefore receives a random copy of the signal transmitted by the source. Fading results in an inherent unreliability of the wireless channel and, in turn, this unreliability is the fundamental limitation to increasing data rates. Methods to raise the reliability of the wireless channel are thus a main concern. A most used method successful in considering the poor effects of channel fading is diversity. Diversity can be employed in the temporal dimension by the utilization of channel coding and an interleaver, the frequency dimension through frequency hopping, or in the spatial dimension through multiple antennas. In all cases, diversity allows a user to average the good and bad fades such that a user sees a channel with lower variance. This paper focuses on exploiting the spatial diversity available in distributed, mesh-like, networks.

In distributed wireless systems, cooperative diversity [9] and relaying [8] can harness the advantages of multiple antennas without using multiple antennas on receivers and transmitters. For practical distributed networks this is motivated by the need for simple, inexpensive nodes with limited processing power and a single receive antenna. Additional antennas, if available, can be used to provide further performance gains.

Most available research on cooperative diversity has focused on a coherent addition of multiple independently-faded copies of the signal. The challenge in such a system is presented by the difficulty of sending multiple copies of the same data from distributed nodes.

This paper provides the techniques for using the multiple relays [17] in wireless communication. The proposed technique will make use of multiple hops [10] for further improvement in wireless communication. The cooperation [4, $5]$ is combined a routing scheme [13, 14] for using multiple hops. The routing algorithm used in this paper is optimized link state routing.

\section{RELATED WORKS}

Aria et al., [1] proposed grouping and partner selection in cooperative [16] wireless networks. Different results to date have illustrated the merits of one or more relay nodes supporting transmissions in a wireless network. In several practical situations, every node in the network is not simultaneously concerned in all transmission; therefore, protocols are required to form groups or subsets of nodes for the purposes of cooperation. This paper consider this difficulty in the circumstance of regenerative nodes and nonaltruistic cooperation (every nodes have data of their own to broadcast). For a network-wide diversity merit, the protocol must offer each transmitting node with enough "partners" that 
can decode its message with high-enough probability. By considering that the nodes cannot communicate their control decisions (distributed scenario), and that all node chooses to help $\mathrm{n}$ other nodes, this paper point out a simple, static selection strategy that guarantees diversity $n+1$ for all transmissions. The author then taken in consideration of centralized control strategies and study the additional gains that arise from a central control, under various amounts of information being available to the central controller.

Chu et al., [2] provided relay selection technique for lowcomplexity coded cooperation.This paper presents relay selection and selection diversity for coded cooperation in wireless sensor networks, with complexity attribute for the sensor nodes. In earlier methods, a relaying technique based on repeat-accumulate (RA) codes was introduced, where it was imagined that the relay does not carry out decoding and simply uses demodulated bits to form codewords. On the other hand, in a network setting with multiple potential relays where relays do not decode the source transmission, it is not noticeable how to choose the best relay. The best choice involves determining the most excellent relay possibly using density evolution, but is quite complex and time-consuming. It is exposed here that the mutual information of the equivalent relay channel, which is much easier than using DE, is a good selection heuristic. With unexpectedly poor performance when a naive selection technique is used, the significance of a good relay selection scheme is emphasized.

Yu et al., [3] put forth a novel trust-based cooperative node selection scheme in wireless sensor networks. For communication among the cluster head node $(\mathrm{CHN})$ and the distant data fusion center (DFC) with the cooperation of intracluster nodes in wireless sensor networks (WSNs), reliable handling of packets by the cooperative node $(\mathrm{CN})$ is difficult to ensure. In this paper, the author proposes a new $\mathrm{CN}$ selection technique based on trust for WSNs. This technique can select one of high trust nodes to support in the transmission of $\mathrm{CHN}$, which aims to advance the transmission performance over cooperative communication in the presence of potential malicious nodes. Initially, this paper set up a trust model to get better the reliability of transmission by $\mathrm{CN}$ in WSNs, which is based on both the direct trust and the indirect trust of nodes. Then the CHN chooses a node based on the projected trust model to aid the $\mathrm{CHN}$ in transmission. Experimental results indicate that the proposed technique can diminish the impact of the possible malicious nodes on transmission performance and the simulation result also indicates the efficiency of the proposed technique.

\section{METHODOLOGY}

This section provides the scenario of multi-source parallelrelay networks, where data is transmitted from the source to the destination through multiple hops. In this context, this paper proposes Selection Cooperation [11]. A parallel-relay network, though, is a simplified scenario which provides insights about the nature of protocols. On the other hand, it is not a very good model for a practical network. Practical networks will almost certainly involve some communications over multiple hops, implying that relays will have the ability to communicate with one another.

To bridge the gap between parallel-relay and generalizedrelay networks, this paper considers multihop networks, where each relay receives information from an adjacent relay. It is observed that in a multi-hop setting, routing, when implemented according to instantaneous channel conditions, can be interpreted as a version of cooperative diversity. Within a single hop, selection cooperation differs from optimal routing only in the presence of a source-destination link (which provides for one additional diversity path). This observation naturally leads to the question: if routing is a version of cooperation, what is the role of cooperation diversity in a system where routing is adapted to changing channel conditions? The answer to this question is not immediately obvious, since the implementation of cooperation on top of a scheme is suggested that is already cooperative in nature (adaptive channel routing). For the sake of clarity, it is point out here that any future reference to "routing" will refer to "channel adaptive routing", i.e., routing based on instantaneous channel conditions.

To address this question, we focus on the problem of combining routing and cooperative diversity in a multi-hop mesh network with static nodes. In this paper, we accomplish this combining by allowing cooperative diversity on each link between two adjacent relays. We note, however, that this strategy is highly sub-optimal, as this paper does not attempt to propose new routing algorithms; its main goal is simply to offer insights into the effect of cooperation when applied in a straightforward manner on top of a routing algorithm. The insight is the following: cooperative diversity does not provide any rate gains over an optimal routing algorithm. By decreasing the amount of resulting hops, these rate gains can only be achieved when cooperative diversity is incorporated into the route selection algorithm. The paper thus underscores the importance of integrating routing and the selective use of cooperative diversity.

\subsection{Network Model}

The distributed system considered here is shown in figure 1, consisting of a source $\mathrm{S}$, destination $\mathrm{D}$, and NM stationary nodes aligned in a uniformly placed grid. The distance between a node and its four nearest neighbors is $d$. This simple structure approximately models mesh and other networks where nodes are static with known locations.

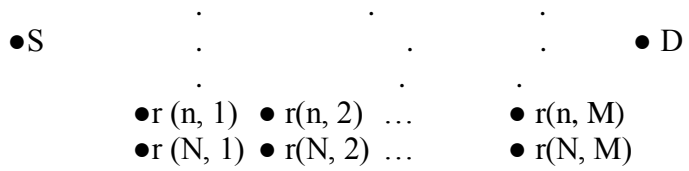

Figure 1: N X M Network Layout. $\mathrm{r}_{(\mathrm{x}, \mathrm{y})}$ denotes a node in position $(\mathrm{x}, \mathrm{y})$. The horizontal and vertical distance between each node is $\mathrm{d}$. 
The channel between nodes in positions ( $\mathrm{i}, \mathrm{j})$ and $(\mathrm{k}, \mathrm{l}), \mathrm{a}_{(\mathrm{i}, \mathrm{j})-(\mathrm{k} \text {, }}$ l), is modelled as flat and slowly-fading Rayleigh with variance $1 / \lambda_{(\mathrm{i}, \mathrm{j})-(\mathrm{k}, \mathrm{l})} ;\left|\mathrm{a}_{(\mathrm{i}, \mathrm{j})-(\mathrm{k}, \mathrm{l})}\right|^{2}$ is exponential with parameter $\lambda_{(\mathrm{i}, \mathrm{j})-(\mathrm{k}, \mathrm{l})}$. This channel is independent of all other channels between remaining nodes. $\lambda_{(i, j)-(k, l)}$, which is inversely proposal to the average channel power, is a function of internode distance, $d_{(i, j)-(k, ~ l)}$, through the attenuation exponent $p_{a}$,

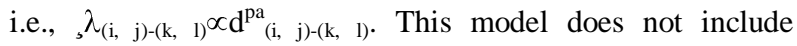
shadowing, although this can easily be incorporated. Note that assuming static nodes and very slowly-fading channels is crucial to the discussion of routing protocols which adapt to changing channels conditions since an adaptation is only possible if the channels are changing slower than the possible rate of adaptation.

A source node $\mathrm{S}$ broadcast data to destination node $\mathrm{D}$ with the help of the NM nodes in the grid. The data can be routed from the source to destination over multiple hops, and the nodes may use Selection Cooperative within each hop. The communication between the source and destination is considered as a flow. Figure 2 illustrates a flow over three hops: cooperative diversity is used in the first and third hops only.

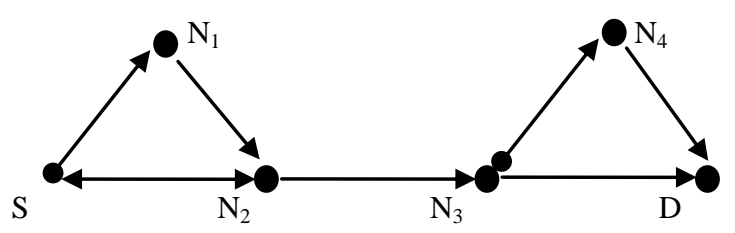

Figure 2: Illustrating multi-hop communications with cooperative diversity.

\subsection{Multiple Accesses}

Transmissions between nodes are accomplished using orthogonal time-slots; since in this thesis we consider a bandwidth-limited network, each transmission results in a bandwidth penalty, i.e., the amount of information communicated is inversely proportional to the number of hops. In particular, we model the multihop rate loss as follows. The source transmits data to the destination using a time slot of duration normalized to 1 . The half-duplex constraint precludes nodes from transmitting on the same channel simultaneously; in a flow with $\mathrm{N}_{\mathrm{h}}$ hops, therefore, each hop uses a time slot of duration $\left(1 / \mathrm{N}_{\mathrm{h}}\right)$. This model explicitly accounts for the increased traffic due to splitting large hops or implementing cooperative diversity.

The nodes considered are stationary and each node transmits at its maximum available power of $\mathrm{P}$ Watts. Furthermore, the MAC protocol eliminates the need for strict power control, and the total power is also unconstrained: a flow through $\mathrm{N}_{\mathrm{h}}$ hops, for example, would consume $\mathrm{N}_{\mathrm{h}} \mathrm{P}$ Watts. Although it might at first glance seem unfair to compare two scenarios with different hop numbers, each using different power levels, we argue that such a scenario is applicable to systems with stationary nodes with dedicated power supplies.

\subsection{Routing and Cooperation Algorithms}

The various schemes that are needed to transmit information from the source $S$ to the destination $D$ are discussed in this section. All the nodes use DF repetition coding. It is to be noted that, in most of these methods, the transmitted information requires knowledge of forward channel power, which requires either centralization or a scheme to distribute channel information throughout the network. It is assumed that, since the nodes are stationary, the channel is changing slowly enough to allow for such a distribution of information. Simultaneously, it is stressed that the approach serve just as performance bounds but their implementation requires the development of more efficient routing algorithms.

The channel between nodes (i, j) and (k, l), $\left|a_{(i, j)-(k, l)}\right|$ implies a mutual information between the nodes, $\mathrm{I}_{(\mathrm{i}, \mathrm{j})-(\mathrm{k}, \mathrm{l})}=\log _{2}(1+$ $\left.\operatorname{SNR}\left|a_{(i, j)-(k, ~ l)}\right|^{2}\right)$. Signal to Noise Ratio is defined as SNR = P/ $\mathrm{N}_{0} \mathrm{~W}$, in which $\mathrm{P}$, represents the power transmitted by each node, $\mathrm{W}$ represent the bandwidth available for each transmission, and $\mathrm{N}_{0}$ is the noise power spectral density. To be sustainable, the data rate over this channel, $R_{(i, j)-(k, l)}$ must be less than the mutual information.

\subsection{2-Hop Smart Cooperation}

This algorithm always uses two hops. This is the simplest approach by combining Smart Cooperation and multi-hop communications. The source transmits to the intermediary node $n_{I}$ with index $(\lfloor N / 2\rfloor,\lfloor M / 2\rfloor)$, where $\lfloor\cdot$ indicates rounding to the nearest lower integer. In order to increase the rate $\mathrm{R}_{\mathrm{nI}-\mathrm{D}}$ between the source and $\mathrm{n}_{\mathrm{I}}$, and the rate $\mathrm{R}_{\mathrm{nI}-\mathrm{D}}$ between $n_{I}$ and the destination, 2-Hop Smart Cooperation approach is implemented. For both hops, all (NM/ 2)-1 are used as potential cooperative nodes (the intermediary node is precluded from cooperating with itself). The final rate, $\mathrm{R}_{\mathrm{SC}-2}$ is the minimum of the rates achieved on both hops, $\min \left\{R_{\mathrm{S}-\mathrm{nI}}\right.$, $\mathrm{R}_{\mathrm{nI}-\mathrm{D}}$ \}. The two-hop transmission implies that transmission on each hop occurs using a time-slot of duration 1/2.

\subsection{Dynamic Routing}

Dynamic routing searches for the rate-maximizing path between the source and destination. In order to ensure forward progress, the maximum number of hops is constrained to $\mathrm{M}+$ 1 , which means that, backward hopping or along the same vertical axis is not possible. The rate $R_{D R}$ is the maximum of all rates attained using any number of hops less than $\mathrm{M}+1$. For a specific $\mathrm{N}_{\mathrm{h}}$-hop flow, the flow rate is $\left(1 / \mathrm{N}_{\mathrm{h}}\right)$-th of the minimum of the mutual information achieved on each hop. The $\left(1 / \mathrm{N}_{\mathrm{h}}\right)$ factor accounts for the half-duplex constraint and penalizes multiple-hop routes. The algorithm can be summarized as follows:

1. Determine the one-hop rate: $\mathrm{R}_{1}=\mathrm{I}_{\mathrm{SD}}$.

2. For $2 \leq N_{h} \leq(M+1)$, determine the rate achieved on each of the possible $\mathrm{N}_{\mathrm{h}}$-hop flows. This rate is the maximum of the minimum of the $\mathrm{N}_{\mathrm{h}}$ mutual information terms: 


$$
\begin{gathered}
R_{N_{h}}=\operatorname{maxmin} \frac{1}{N_{h}}\left\{I_{S-(i, j)}, I_{(i, j)-(k . l), \ldots . .,} I_{(m, n)-D}\right\} \\
1 \leq(i, k, \ldots . . m) \leq N, 1 \leq j<l, \ldots . .<n \leq M
\end{gathered}
$$

Where the optimization is over indices (i, k, .., m) and (j, l, $\ldots, n$ ), i.e., for each $\mathrm{N}_{\mathrm{h}}$, the maximization is over all possible flows consisting of $\mathrm{N}_{h}$ hops, and thus over all node-indices. The strict inequality in the y-coordinate ensures forward progress by eliminating the possibility of routing backwards or along a vertical line (note that in our definition, the S - D link is along the y-direction).

3. Find the maximum over all the flows:

$$
R_{D R}=\max \left\{R_{1}, R_{2}, \ldots . ., R_{M+1}\right\}
$$

\subsection{Dynamic Routing with Cooperation}

This approach sequentially combines Dynamic Routing and Smart Cooperation. Dynamic Routing approach is used to choose the optimal dynamic route. Cooperation is used to increase the rate along this path with this route, i.e.,Smart Cooperation is applied between the nodes chosen by the Dynamic Routing algorithm.

\subsection{Dynamic Cooperative Routing}

This routing simultaneously integrates Dynamic Routing with Cooperation: the optimal path is chosen together with the cooperative partners. As in Dynamic Routing, the Dynamic Cooperative Rate RDCR is the maximum of the rates achieved with different hops:

$$
\mathrm{R}_{\mathrm{DCR}}=\max \left\{\mathrm{R}_{1}, \mathrm{R}_{2}, \ldots ., \mathrm{R}_{\mathrm{M}+1}\right\}
$$

Unlike Dynamic Routing, however, $\mathrm{R}_{\mathrm{DCR}}$ is achieved with cooperation potentially included in each of the $\mathrm{N}_{\mathrm{h}}$ hops, i.e., for each possible combination of hops, the algorithm implements Smart Cooperation along each hop. This algorithm is very similar to Dynamic Routing except for the cooperation included in the search for optimal route. An outline of this algorithm is given below:

1. Perform the Smart Cooperation algorithm. The result is $\mathrm{R}_{1}$, the maximum rate achieved with one hop.

2. For $N_{h} \leq M+1$, find a set of all possible $N_{h}$-hop routes between source and destination. For each route, perform Smart Cooperation along all hops, i.e., determine if any cooperating partner can increase the rate along any of the $\mathrm{N}_{\mathrm{h}}$ hops. $R_{N_{h}}$, the rate of a $\mathrm{N}_{\mathrm{h}}$-hop route, is the minimum rate along each of the $\mathrm{N}_{\mathrm{h}}$ hops (including a factor of $1 / \mathrm{N}_{\mathrm{h}}$ for bandwidth expansion).

3. The final rate $R_{D C R}$ is the maximum of the rates obtained using all possible routes and cooperating partners.

Note that dynamic cooperative routing is an extremely complex scheme with large computation overhead.

In order to decrease algorithmic complexity, the routing algorithm is implemented using Dijkstra's algorithm. The metrics used by these approaches are not isotonic and thus it does not guarantee optimal paths [97]. The main aim is to investigate the relationship between routing and cooperation and not to determine the optimal routing algorithm; the suboptimality of this implementation is not of great importance.

To overcome these problems, the routing can be performed with the help of Optimized Link State Routing (OLSR).

\subsection{Optimized Link State Routing}

The protocol inherits the stability of the link state algorithm. It has the benefit of having the routes immediately available due to its proactive nature. In a pure link state protocol, all the links with neighbor nodes are declared and are flooded in the entire network. Firstly, the size of control packets is minimized: instead of all links, it declares only a subset of links with its neighbors who are its multipoint relay selectors. Next, it minimizes flooding of this control traffic by with the help of only the selected nodes, called multipoint relays, to spread its messages in the network. Only the multipoint relays of a node retransmit its transmitted messages. This method considerably decreases the number of retransmissions in a flooding or broadcast procedure.

\subsection{Protocol functioning}

\subsubsection{Neighbor sensing}

Every node should detect the neighbor nodes through which it has a direct and bi-directional link. The difficulties over radio propagation may create some links uni-directional. As a result, all links should be checked in both directions in order to be considered valid.

To accomplish this, each node periodically broadcasts its messages, containing the information about its neighbors and their link status. These control messages are transmitted in the broadcast mode. These are received by all one-hop neighbors, but they are not relayed to further nodes. A message contains:

- The list of addresses of the neighbors to which there exists a valid bi-directional link

- The list of addresses of the neighbors which are heard by this node (a message has been received) but the link is not yet validated as bi-directional if a node finds its own address in a message, it considers the link to the sender node as bi-directional.

These messages permit every node to be trained with the information of its neighbors up to two hops. On the basis of this knowledge, all nodes perform the selection of its multipoint relays. These selected multipoint relays are represented in the messages with the link status MPR. On the reception of messages, all node can build its MPR Selector table with the nodes that have selected it as a multipoint relay in the neighbor table, each node records the information about its one hop neighbors, the status of the link with these neighbors, and a list of two hop neighbors that these one hop neighbors give access to. The link status can be unidirectional, bi-directional or MPR. The link status as MPR implies that the link with the neighbor node is bi-directional AND that node is also selected as a multipoint relay by this 
local node. Each entry in the neighbor table has an associated holding time, upon, expiry of which it is no longer valid and hence removed.

The neighbor table also contains a sequence number value which specifies the most recent MPR set that the local node keeping this neighbor table has selected. Every time a node selects or updates its MPR set, this sequence number is incremented to a higher value.

\subsubsection{Multipoint relay selection}

Every node of the network chooses separately its own set of multipoint relays. The MPR set is computed in a way to include a subset of one hop neighbors which covers all the two hop neighbors, i.e., the union of the neighbor sets of every MPRs contains the complete two hop neighbor set. For building the list of the two hop nodes from a provided node, it suffices to follow the list of bidirectional link nodes found in the messages received by this node The MPR set is necessarily not be optimal, though it must be small enough to attain the merits of multipoint relays. By default, the multipoint relay set can agree with the whole neighbor set. This will be the case at network initialization.

Multipoint relays of a given node are declared in the subsequent message transmitted by this node, so that the information arrives at the multipoint, relays themselves. The multipoint relay set is re-calculated when:

- A modification in the neighborhood is identified when either a bi-directional link with a neighbor is failed, or a new neighbor with a bi-directional link is added; or

- A modification in the two-hop neighbor set with bidirectional link is identified.

With information gathered from the messages, every node also built its MPR Selector table, in which it places the addresses of its one hop neighbor nodes which has chosen it as a multipoint relay along with the equivalent MPR sequence number of that neighbor node. A sequence number is also linked to the MPR Selector table that indicates that the MPR Selector table is most recently altered with that sequence number. A node updates its MPR Selector set based on the information it receives in the messages, and increment this sequence number on every modification.

\section{EXPERIMENTAL RESULTS}

In this section, the simulation result for the proposed method is presented. The outage probability is considered for the comparison. The proposed method is compared with Distributed Space-Time Coding (DSTC) [6, 7]. With equal average channel powers with parameter $\lambda$, the probability of outage of the proposed selection scheme in the high-SNR regime is

$$
\begin{array}{r}
\operatorname{Pr}\left[I_{\text {simple-sel }}<R\right] \\
=\left[\left(2^{2 R}-1\right)\right. \\
/ S N R]^{m} \lambda^{m+1} \sum_{|D(s)|} \frac{K_{|D(s)|}(m)}{|D(s)|+1}
\end{array}
$$

Where,

$$
K_{|D(s)|}(m)=\sum_{N=1}^{m-1} \frac{\frac{N^{|D(s)|}}{(m-1) ! N !(m-1)^{N}}}{\sum_{k=1}^{m-1} \frac{1}{(m-k) !(m-1)^{k}}}
$$

The analytical results are obtained by calculating the above equation for increasing network sizes with $\lambda_{\mathrm{ij}}=1$ and $\mathrm{R}=1$ $\mathrm{b} / \mathrm{s} / \mathrm{Hz}$.

The approximation assumes that the decoding set contains $\mathrm{m}$ - 1 node for every source node, i.e., that every potential relay node correctly decodes the information from every source.

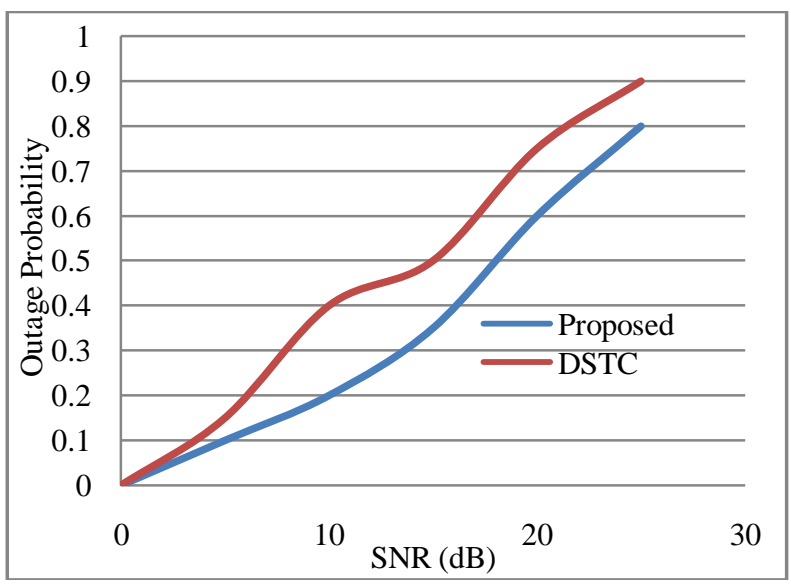

Figure 3: Outage Probability Comparison for various SNR value

Figure 3 shows the comparison of the proposed technique with DSTC in terms of outage probability for various SNR values. As observed from figure 3, the outage probability for the proposed method is 0.1 for the proposed method and 0.15 for the DCTC technique for SNR value of 5. For the SNR value 10 , the outage probability is 0.4 for DSTC, whereas, only 0.2 for proposed method. The outage probability is 0.5 for DSTC and only 0.35 for SNR value 15 . For the SNR value 12 , the outage probability is 0.75 for DSTC, whereas, only 0.6 for proposed method. The outage probability is 0.9 for DSTC and only 0.8 for SNR value 25 . When the overall outage probability is considered, the proposed method outperforms the DSTC method in for value of SNR value.

\section{CONCLUSION}

In this paper, the novel node selection technique is proposed in cooperative wireless networks. The usage of proposed technique in single-hop network transmission with multiple relays between the source and destination will produce better results comparing to the existing techniques such as distributed space-time codes. In order to further improve the performance in wireless communication, the multiple-hops can be used instead of single-hop. The combination of cooperation and channel-adaptive routing is implemented for wireless communication in case of multiple-hop transmission. But it will be very complex and includes large computation for using the routing technique. In order to reduce these 
complexities, optimized link state routing technique is implemented in this paper. The simulation result shows that the propose technique outperforms the existing techniques such as distributed space-time codes.

\section{REFERENCES}

[1] Aria Nosratinia and Todd E. Hunter, "Grouping and partner selection in cooperative wireless networks", IEEE Journal on Selected Areas in Communications, Vol.25, No.2, 2007.

[2] Chu. J.P.K, Adve. R.S and Eckford. A.W, "Relay Selection for Low-Complexity Coded Cooperation", IEEE Global elecommunications Conference, 2007.

[3] Yu Zhang, Qiongli Liu, YuemingCai, Weiwei Yang and YouyunXu, "A Novel Trust-based Cooperative Node Selection Scheme in Wireless Sensor Networks", WRI International Conference on Communications and Mobile Computing, Vol. 2, 2009.

[4] J. N. Laneman, D. N. C. Tse and G. W. Wornell, "Cooperative diversity in wireless networks: Efficient protocols and outage behavior", IEEE Transactions on Information Theory, vol. 50, pp. 3062 - 3080, December 2004.

[5] P. Herhold, E. Zimmermann, and G. Fettweis, "Cooperative multihop transmission in wiresless networks", Computer Networks Journal, 2004.

[6] S. Barbarossa and G. Scutari, "Distributed space-time coding for multihop networks", in Proceedings of IEEE International Conference on Communications, ICC 2004, June 2004.

[7] M. Uysal and O. Canpolat, "On the distributed spacetime signal design for a large number of relay terminals" in Proceedings of IEEE Wireless Communications and Networking Conference, March 2005.

[8] S. Hares, H. Yanikomeroglu, and B. Hashem, "A relaying algorithm for multihop TDMA TDD networks using diversity", in Proceedings of IEEE Vehicular Technology Conference, October 2003.
[9] F. H. P. Fitzek and M. D. Katz, "Cooperation in Wireless Networks: Principles and Applications. Springer, 2006.

[10] J. Boyer, D. Falconer, and H. Yanikomeroglu, "Multihop diversity in wireless relaying channels", IEEE Trans. Commun., vol. 52, pp. 1820 - 1830, October 2004.

[11] J. Boyer, D. Falconer, and H. Yanikomeroglu, "Cooperative connectivity models for wireless relay networks," IEEE Trans. Wireless Commun., vol. 6, no. 10, pp. 1992-2000, June 2007.

[12] Z. Yang and A. Host-Madsen, "Routing and power allocation in asynchronous Gaussian multiple-relay channels," EURASIP Journal on Wireless Communications and Networking, 2006.

[13] S.H. Chen, U. Mitra, and B. Krishnamachari, "Cooperative communication and routing over fading channels in wireless sensor network," in Proc. of the IEEE International Conference on Wireless Networks,Communications, and Mobile Computing (Wireless-Com), June 2005.

[14] X. Fang, T. Hui, Z. Ping, and Y. Ning, "Cooperative routing strategies in Ad Hoc networks", in Proc. of the IEEE Vehicular Technology Conference (VTC-Spring 2005), June 2005.

[15] A. Khandani, J. Abounadi, E. Modiano, and L. Zhang, "Cooperative routing in wireless networks," in Proc. of Allerton Conference on Communications, Control and Computing, October 2006

[16]L. Ong and M. Motani, "Optimal routing for decode-andforward based cooperation in wireless networks," in Proc. of the IEEE Conference on Sensor, Mesh and Ad Hoc Communications and Networks (SECON), June 2007, san Diego.

[17]J. Chu, R. S. Adve, and A. Eckford, "Relay selection for low-complexity coded cooperation," in Proc. of IEEE Global Telecommunications Conference, December 2007. 